This item was submitted to Loughborough's Research Repository by the author.

Items in Figshare are protected by copyright, with all rights reserved, unless otherwise indicated.

\title{
Surface and interstitial transition barriers in rutile (110) surface growth
}

PLEASE CITE THE PUBLISHED VERSION

http://dx.doi.org/10.1103/PhysRevB.80.235308

\section{PUBLISHER}

(c) The American Physical Society

VERSION

VoR (Version of Record)

LICENCE

CC BY-NC-ND 4.0

\section{REPOSITORY RECORD}

Sanville, E., Louis J. Vernon, Steven D. Kenny, Roger Smith, Y. Moghaddam, C. Browne, and P. Mulheran. 2019. "Surface and Interstitial Transition Barriers in Rutile (110) Surface Growth". figshare.

https://hdl.handle.net/2134/11627. 
This item was submitted to Loughborough's Institutional Repository (https://dspace.lboro.ac.uk/) by the author and is made available under the following Creative Commons Licence conditions.

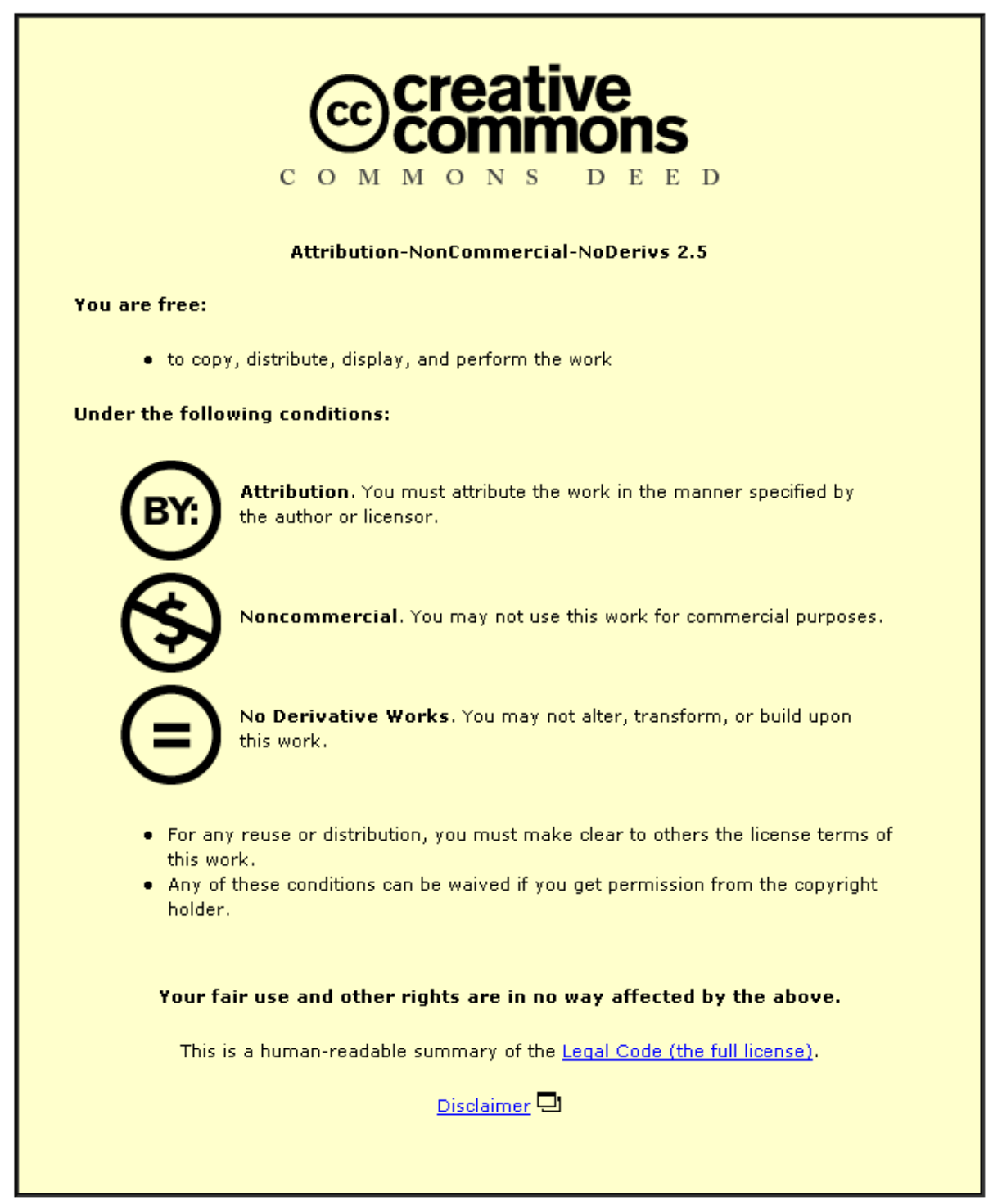

For the full text of this licence, please go to: http://creativecommons.org/licenses/by-nc-nd/2.5/ 


\title{
Surface and interstitial transition barriers in rutile (110) surface growth
}

\author{
E. J. Sanville, ${ }^{1, *}$ L. J. Vernon, ${ }^{1}$ S. D. Kenny, ${ }^{1}$ R. Smith,${ }^{1}$ Y. Moghaddam,${ }^{2, \dagger}$ C. Browne, ${ }^{2}$ and P. Mulheran ${ }^{3, *}$ \\ ${ }^{1}$ Department of Mathematical Sciences, Loughborough University, Loughborough LE11 3TU, United Kingdom \\ ${ }^{2}$ Department of Physics, University of Reading, Whiteknights, Reading RG6 6AF, United Kingdom \\ ${ }^{3}$ Department of Chemical and Process Engineering, University of Strathclyde, James Weir Building, 75 Montrose Street, Glasgow G1 \\ IXJ, United Kingdom
}

(Received 24 November 2008; revised manuscript received 19 March 2009; published 7 December 2009)

\begin{abstract}
We present calculated surface and interstitial transition barriers for $\mathrm{Ti}, \mathrm{O}, \mathrm{O}_{2}, \mathrm{TiO}$, and $\mathrm{TiO}_{2}$ atoms and clusters at the rutile (110) surface. Defect structures involving these small clusters, including adcluster and interstitial binding sites, were calculated by energy minimization using density-functional theory (DFT). Transition energies between these defect sites were calculated using the NEB method. Additionally, a modified SMB-Q charge equilibration empirical potential and a fixed-charge empirical potential were used for a comparison of the transition energy barriers. Barriers of 1.2-3.5 eV were found for all studied small cluster transitions upon the surface except for transitions involving $\mathrm{O}_{2}$. By contrast, the $\mathrm{O}_{2}$ diffusion barriers along the [001] direction upon the surface are only $0.13 \mathrm{eV}$. The QEq charge equilibration model gave mixed agreement with the DFT calculations, with the barriers ranging between 0.8 and $5.8 \mathrm{eV}$.
\end{abstract}

DOI: 10.1103/PhysRevB.80.235308

PACS number(s): 68.43.Jk, 68.43.Mn, 68.35.Fx, 68.55.ag

\section{INTRODUCTION}

$\mathrm{TiO}_{2}$ is a wide band-gap metal oxide with a large number of uses. ${ }^{1}$ Under normal conditions, its most stable form is the rutile polymorph. In addition to being useful as an optical coating, rutile can also be found as a substrate in Graetzeltype solar cells ${ }^{2}$ and in various photocatalytic applications. ${ }^{3-5}$ Modern industrial windows and windshields are typically manufactured with optical and mechanical coatings composed of several oxide thin-film layers deposited on a glass or polymer surface. ${ }^{6}$ These thin films include $\mathrm{ZnO}, \mathrm{Ag}$, and the anatase and rutile polymorphs of $\mathrm{TiO}_{2}$. Each layer performs a different function, for example, to obtain antireflection or antiscratching properties. One important function of the anatase and rutile layers is to provide "self-cleaning" properties to the glass surface. ${ }^{7,8}$ This interesting property is derived from the catalytic ability of anatase in particular to degrade adsorbed hydrocarbon molecules combined with the photoinduced hydrophilicity of the surface, which allows wetting of the surface by a water layer. This hydrophilicity prevents water droplet formation providing an antifogging property to the glass. ${ }^{8}$ Rutile thin films have a high refractive index ${ }^{9}$ and are therefore useful for providing antireflective properties to the glass. In order to achieve high deposition rates and smooth surfaces, functional coatings are typically deposited via magnetron sputtering. ${ }^{8}$ Currently we lack a good theoretical understanding of the main growth mechanism of rutile thin films by deposition of small titaniumoxygen clusters. In addition most dynamical growth models use empirical potentials since the calculations are generally too lengthy for $a b$ initio techniques. However, $a b$ initio methods can be used in a more limited way to determine the main transition barriers and bonding sites for the clusters on a surface. If the general trends of the $a b$ initio calculations are reflected by the empirical models this gives some confidence that the empirical models can be used for the more time-consuming dynamical growth simulations. This is one of the main purposes behind the work described here.
The (110) rutile surface is the most thermodynamically stable surface ${ }^{10}$ of $\mathrm{TiO}_{2}$. The unreconstructed surface has typically been examined in prior literature. This surface is composed of a series of parallel two-coordinated oxygen rows separated by "trenches." Along the two-coordinated oxygen rows, beneath and between the two-coordinated oxygen atoms, are a series of six-coordinated surface titanium atoms. Although these titanium atoms are fully coordinated, they are close enough to the surface that they have important effects on the surface chemistry of adsorption. The trenches are nearly flat areas of the surface that are composed of a network of five-coordinated surface titanium atoms and three-coordinated surface oxygen atoms.

The kinetics of rutile (110) surface growth has been studied via variable-temperature scanning tunneling microscopy. ${ }^{11,12}$ The observations showed that the rutile (110) surface grows by combination of gas-phase oxygen with mobile interstitial $\mathrm{Ti}^{3+}$ ions from the bulk. The diffusion and reaction of titanium interstitials with adsorbed oxygen have been examined theoretically, ${ }^{13}$ and it was found that the energy barriers for $\mathrm{Ti}$ interstitial diffusion and reaction were 0.75 and $1.2 \mathrm{eV}$, respectively. It is our goal to examine theoretically the detailed growth mechanism of the rutile (110) surface, and in this report on the process we present a systematic examination of various small cluster transition barriers upon the rutile (110) surface and within the first subsurface layer.

\section{METHODOLOGY}

Three different methodologies were used for the calculations reported here. The first method is a quantummechanical $a b$ initio approach while the other two are a fixed-charge and a variable charge empirical potential model. Transition pathways were located using the nudged elastic band (NEB) technique ${ }^{14,15}$ and the climbing image nudged elastic band technique. ${ }^{16}$

The $a b$ initio calculations were performed using the 
PLATO (Package for Linear Combination of Atomic Orbitals) suite of programs. ${ }^{17}$ During testing, it was found that the local-density approximation (LDA) resulted in superior surface energy predictions than the available Perdew-BurkeErnzerhof generalized gradient approximation functional. As a result, these were done using the LDA functional, with pseudopotentials ${ }^{18}$ and a triple numeric set of atom-centered basis functions with double polarization. The calculations included semicore electrons on the titanium atoms. In total, the titanium basis set was composed of four $s$ functions, three sets of $p$ functions, and three sets of $d$ functions. These atomiclike basis functions were derived from atomic SCF calculations for neutral $\mathrm{Ti}$, as well as $\mathrm{Ti}^{2+}$ and $\mathrm{Ti}^{4+}$. The oxygen basis set was composed of three $s$ functions, three sets of $p$ functions, and two sets of $d$ functions derived from atomic SCF calculations for neutral $\mathrm{O}, \mathrm{O}^{2+}$, and $\mathrm{O}^{4+}$.

The calculations were carried out using periodic boundary conditions. The supercell contained a slab of rutile with two unreconstructed (110) surfaces. The slab had dimensions of $4 \times 1 \times 2$ tetragonal (110) surface unit cells for a total of 96 atoms. The bottom half of the atoms in the cell were held fixed as this makes the surface energy converge quicker. Exhaustive tests were carried out both changing the surface area and depth until the optimum size was found. The tests showed that the results converged to within $5 \%$ with respect to both slab thickness and lateral dimension. A MonkhorstPack mesh of $k$ points was used of dimensions $1 \times 2 \times 1$.

These $a b$ initio calculations were compared to calculations using two empirical potentials. This was done in order to verify and improve the accuracy of these empirical potentials for potential use in future molecular-dynamics (MD) simulations. It would be desirable to have the ability to model accurately and cheaply sputter deposition by simulating cluster impact processes using an empirical potential. Most of the empirical potential calculations were performed using the $\mathrm{QEq}$ variable charge equilibration scheme. ${ }^{19-22}$ This potential combines the QEq charge equilibration scheme originally proposed by Rappe and Goddard ${ }^{23}$ with short-range potentials for the Ti-O and O-O interactions. The QEq component of the potential adjusts the ionic charges in order to minimize the sum of the ionic self-energies and a screened Coulombic energy. The ionic self-energies are quadratic with respect to the ionic charge with parameters related to the electronegativity and electronic hardness of the element. The Ti-O short-range interaction is based on the second moment approximation of the tight-binding approach, which helps treat the mixed ionocovalent character of the metal-oxygen bonds within insulating oxides. ${ }^{24}$

The QEq atomic charges were calculated by an iterative minimization of the QEq energy with respect to the atomic charges using a conjugate gradient algorithm. The screened Coulombic energies and forces were calculated by using Ewald summation to determine the regular Coulombic energies and forces followed by the subtraction of Coulombic contributions of neighbors within the screening cutoff. The screened Coulombic interactions due to the neighbors, calculated by cubic spline interpolation, were then added to obtain the total screened Coulombic energies and forces. For the calculations involving $\mathrm{TiO}_{2}$ clusters, additional calculations were performed using the fixed-charge empirical model of Matsui and Akaogi. ${ }^{25}$

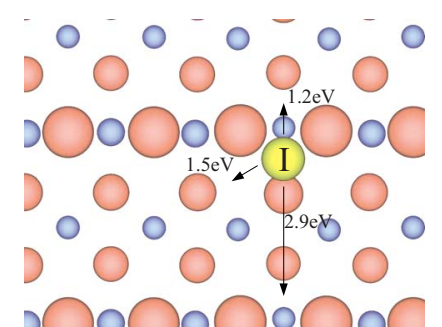

(a)

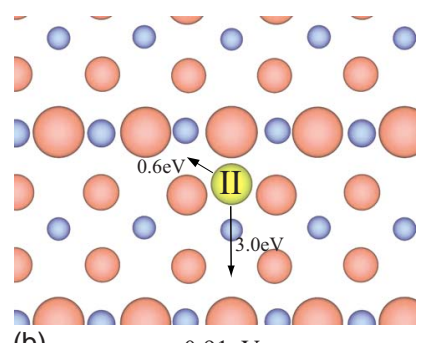

(b)
$0.91 \mathrm{eV}$
FIG. 1. (Color online) The diagrams show the adsorbed titanium atom as shown looking down perpendicular to the (110) surface. The red atoms are oxygen, while the blue atoms are titanium. The horizontal rows of larger oxygen atoms represent the surface rows of two-coordinated oxygen atoms. In (a), the adsorbed titanium atom is shown in the upper hollow site (site I), while in (b), it is shown in the lower hollow site (site II). The arrows represent the various transitions available to the adatom in these adsorption sites. The energies correspond to the DFT activation barriers for each transition.

The nudged elastic band method ${ }^{15}$ was used to calculate each transition pathway as a series of geometric images. In all calculations performed in this paper, a total of ten NEB images were used (including the initial and final images of each transition). The climbing image nudged elastic band method $^{16}$ was used in the case of the calculations involving the empirical potentials, while the regular nudged elastic band method was used for the density-functional theory (DFT) calculations.

\section{RESULTS}

\section{A. Titanium adatom}

There are two adsorption sites for the titanium atom on the rutile (110) surface. The "upper hollow" site in Fig. 1(a) has the adsorbed titanium atom bound to two adjacent twocoordinated oxygen atoms and one three-coordinated oxygen atom. The "lower hollow" site in Fig. 1(b) has the adsorbed titanium atom bound to two three-coordinated oxygen atoms and one two-coordinated oxygen atom. The upper hollow adsorption site was calculated by DFT to have a net binding energy of $-4.95 \mathrm{eV}$ relative to the system composed of a free titanium atom in vacuum plus a perfect rutile (110) surface. The relative energies of these binding sites, as calculated by DFT and the QEq model, are given in Table I. The reason that the $\mathrm{Ti}$ site $\mathrm{II}$ interstitial is higher in energy than the $\mathrm{Ti}$ site I is likely due to the fact that in site II the Ti interstitial bonds to two surface $\mathrm{O}$ atoms, whereas in site I it bonds to only one. The surface $\mathrm{O}$ atoms have less charge, thus, making site I more favorable.

The arrows in Fig. 1 show the calculated transitions with their corresponding DFT activation barriers. The activation barriers as calculated by DFT and the QEq model are given in Table II. There is a short transition from upper hollow to lower hollow, shown in Fig. 1(a), and the profile for this transition is shown in Fig. 2(a). From the upper hollow site, the titanium atom can also hop along the [1필 direction ending at an adjacent upper hollow position. There are two 
TABLE I. The relative energies of each of the binding sites for the $\mathrm{Ti}$ adatom, $\mathrm{O}$ adatom, $\mathrm{TiO}$ adcluster, and $\mathrm{TiO}_{2}$ adcluster as calculated by DFT and the QEq variable charge potential, respectively. For each type of cluster, the energies are given in $\mathrm{eV}$, relative to the lowest-energy binding site.

\begin{tabular}{lccc}
\hline \hline Binding site & $\begin{array}{c}\text { Relative DFT energy } \\
(\mathrm{eV})\end{array}$ & $\begin{array}{c}\text { Relative QEq energy } \\
(\mathrm{eV})\end{array}$ & $\begin{array}{c}\text { Relative fixed-charge energy } \\
(\mathrm{eV})\end{array}$ \\
\hline Ti upper hollow & 1.91 & 2.20 \\
Ti lower hollow & 2.82 & 2.68 \\
Ti-I interstitial & 0.00 & 0.00 & \\
Ti-II interstitial & 0.26 & 0.08 & \\
O-I & 0.00 & & \\
O-II & 1.44 & 0.24 & 0.00 \\
TiO-I & 0.00 & 0.00 & 1.08 \\
TiO-II & 0.61 & 0.77 & \\
TiO-III & 0.85 & 0.56 & \\
TiO-IV $_{\text {TiO }}$-I & 1.32 & 0.00 & \\
$\mathrm{TiO}_{2}$-II & 0.00 & 0.44 & \\
\hline \hline
\end{tabular}

ways this can occur, both of which are illustrated in Fig. 1(a). In the first type of transition $(1.2 \mathrm{eV})$ the titanium atom can hop over the adjacent two-coordinated oxygen row ending at the upper hollow site on the other side of the same row. The second type of upper hollow-upper hollow transition (2.9 $\mathrm{eV}$ ) has the titanium adatom move in the opposite direction toward an adjacent row of two-coordinated oxygen atoms. There is also a corresponding lower hollow-lower hollow transition, where the $\mathrm{Ti}$ in the lower hollow site moves across the trench to the opposite lower hollow site $(3.0 \mathrm{eV})$ shown in Fig. 1(b).

\section{B. Titanium interstitial}

There are two unique interstitial sites for the titanium atom within the first layer beneath the (110) surface both illustrated in Fig. 3. The interstitial titanium atom is six coordinated at both sites, with the neighboring oxygen atoms in an octahedral arrangement around the interstitial titanium. In the first site, Ti-iI, one of the six neighboring oxygen atoms is within the surface layer. In the Ti-iII site, two of the neighboring oxygen atoms are within the surface layer. Within the rutile bulk, these two interstitial sites are symmetrically equivalent, however, here the presence of the surface differentiates them. The relative energies of these binding sites, as calculated by DFT and the QEq model, are given in Table I.

The Ti-iI interstitial can be formed from the upper hollow adatom as shown in Fig. 3. During this process, the adatom pushes the nearest six-coordinated titanium surface atom into the Ti-iI interstitial site. In the process, the adatom moves into the six-coordinated titanium surface site replacing the titanium which has now become the interstitial atom. The Ti-iII interstitial can be formed from the lower hollow adatom, in a similar process to that of Ti-iI, which is also shown in Fig. 3. In this case, the lower hollow adatom pushes the nearest five-coordinated titanium surface atom into a Ti-iII interstitial site while moving into the previously occupied five-coordinated titanium surface site. A Ti-iI interstitial can move into a Ti-iII site by simple displacement along the [0 0 1] direction and vice versa. The energy profile for this transition is shown in Fig. 2(b). The probable reason why the replacement mechanism is lower in energy than direct migration of the $\mathrm{Ti}$ interstitial to the surface is because fewer bonds are broken and there is less strain in the transition.

Table II shows that there is one transition where the QEq model disagrees substantially with the DFT results. This is the case of the adatom Ti moving to an interstitial position via a replacement mechanism, although in both cases the Ti interstitial forms a lower energy structure than the adatom. The QEq model gives a barrier of $0.47 \mathrm{eV}$ compared to 1.76 $\mathrm{eV}$ for DFT for the Ti lower hollow-Ti-iII transition, and $0.85 \mathrm{eV}$ compared to $1.60 \mathrm{eV}$ for DFT. Similar values are also obtained for the direct transition without replacement.

\section{Oxygen adatom}

There are two adsorption sites for an oxygen adatom on the rutile (110) surface. In the first site, O-I, the oxygen atom bonds to a two-coordinated oxygen atom within the surface and forms an adsorbed dioxygen unit. In this adsorption site, shown in Fig. 4(a), the two atoms are then located symmetrically with respect to the $(1 \overline{1} 0)$ plane. This oxygen adatom binding site was calculated to have a net DFT binding energy of $3.30 \mathrm{eV}$ relative to the free atom and perfect surface. The $\mathrm{O}-\mathrm{O}$ distance between the two symmetrically equivalent atoms was calculated to be $1.416 \AA$, which is significantly longer than the $\mathrm{O}_{2}$ bond length of $1.225 \AA$ calculated with the same methodology. A Bader analysis ${ }^{26,27}$ was performed on this system to investigate further the nature of the $\mathrm{O}_{2}$ unit. The results of this investigation are summarized in Table III. The Bader charge on each of the oxygen atoms was found to be $-0.42 e$, with no spin density. The reason for this level of charge on the $\mathrm{O}$ adatom is that when it bonds to a surface $\mathrm{O}$ atom, each $\mathrm{O}$ atom in the dimer forms a bond with the neighboring surface Ti atom. This is also quite different from the 
TABLE II. The calculated energy barriers of each of the transitions. Barriers are given in $\mathrm{eV}$ for the DFT and the QEq potential calculations.

\begin{tabular}{|c|c|c|c|}
\hline \multirow[b]{2}{*}{ Transition } & \multicolumn{2}{|c|}{$\begin{array}{l}\text { Energy barrier } \\
\qquad(\mathrm{eV})\end{array}$} & \multirow[b]{2}{*}{ Fixed charge } \\
\hline & DFT & QEq & \\
\hline Ti upper-lower hollow & 1.52 & 1.11 & \\
\hline Ti lower-upper hollow & 0.61 & 0.63 & \\
\hline Ti upper hollow-iI & 1.60 & 0.85 & \\
\hline Ti iI-upper hollow & 3.51 & 3.05 & \\
\hline Ti lower hollow-iII & 1.76 & 0.47 & \\
\hline Ti iII-lower hollow & 4.36 & 3.08 & \\
\hline Ti iI-iII & 1.23 & 0.75 & \\
\hline Ti ilI-iI & 0.97 & 0.67 & \\
\hline Ti upper hollow-upper hollow a & 1.20 & 1.27 & \\
\hline Ti upper hollow-upper hollow b & 2.90 & 2.71 & \\
\hline Ti lower hollow-lower hollow & 3.00 & 2.92 & \\
\hline O-I to O-I & 2.51 & & \\
\hline O-I to O-II & 2.34 & & \\
\hline O-II to O-I & 0.90 & & \\
\hline O-II to O-II & 0.29 & 0.78 & \\
\hline TiO-I to TiO-Ia & 0.70 & 0.46 & \\
\hline TiO-I to TiO-II & 0.63 & 0.21 & \\
\hline TiO-II to TiO-I & 0.02 & 0.43 & \\
\hline TiO-I to TiO-III & 1.12 & 0.63 & \\
\hline TiO-III to TiO-I & 0.27 & 0.11 & \\
\hline TiO-II to TiO-IIa & 1.34 & 1.08 & \\
\hline TiO-II to TiO-IIb & 3.50 & 3.14 & \\
\hline TiO-II to TiO-IV & 1.48 & 1.01 & \\
\hline TiO-IV to TiO-II & 0.78 & 0.45 & \\
\hline TiO-III to TiO-IV & 0.56 & 0.02 & \\
\hline TiO-IV to TiO-III & 0.01 & 0.31 & \\
\hline $\mathrm{TiO}_{2}-\mathrm{I}$ to $\mathrm{TiO}_{2}-\mathrm{Ia}$ & 3.26 & 2.10 & 2.90 \\
\hline $\mathrm{TiO}_{2}-\mathrm{I}$ to $\mathrm{TiO}_{2}-\mathrm{Ib}$ & 2.96 & 2.01 & 2.60 \\
\hline $\mathrm{TiO}_{2}-\mathrm{I}$ to $\mathrm{TiO}_{2}-\mathrm{Ic}$ & 5.03 & 2.34 & 5.67 \\
\hline $\mathrm{TiO}_{2}-\mathrm{I}$ to $\mathrm{TiO}_{2}-\mathrm{II}$ & 1.27 & 0.51 & 1.57 \\
\hline $\mathrm{TiO}_{2}-\mathrm{II}$ to $\mathrm{TiO}_{2}-\mathrm{I}$ & 0.17 & 0.07 & 0.49 \\
\hline $\mathrm{TiO}_{2}$-II to $\mathrm{TiO}_{2}$-IIa & 1.37 & 0.59 & 0.25 \\
\hline $\mathrm{TiO}_{2}$-II to $\mathrm{TiO}_{2}$-IIb & 3.51 & 0.79 & 0.99 \\
\hline $\mathrm{O}_{2}$-I to $\mathrm{O}_{2}$-Ia & 0.13 & & \\
\hline $\mathrm{O}_{2}-\mathrm{I}$ to $\mathrm{O}_{2}-\mathrm{Ib}$ & 0.11 & & \\
\hline $\mathrm{O}_{2}$-I to $\mathrm{O}_{2}$-Ic & 0.36 & & \\
\hline
\end{tabular}

oxygen atoms of an isolated $\mathrm{O}_{2}$ molecule, which of course have zero charge and an atomic spin of 0.5. Additionally, the Laplacian of the electronic charge density at the bonding critical point was calculated to be 0.24 . Because this is a positive value, this indicates that the O-O bond is actually a "closed-shell" interaction rather than a covalent bond. The Laplacian is -0.33 (negative, indicating a covalent bond) for the dioxygen molecule in vacuum using the same DFT methodology. Because the QEq potential lacks an attractive O-O

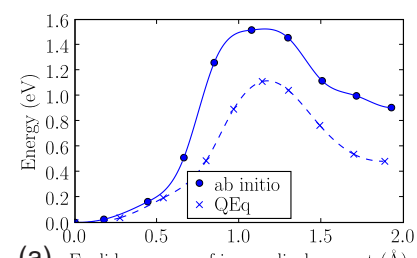

(a) Euclidean norm of image displacement $(\AA)$

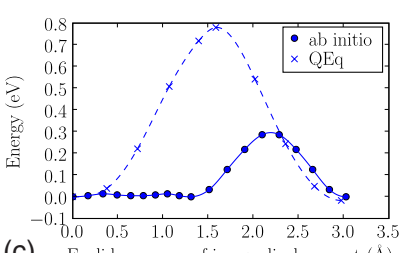

(C) Euclidean norm of image displacement $(\AA)$

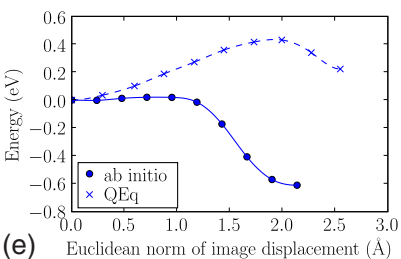

(e) Euclidean norm of image displacement $(\AA)$

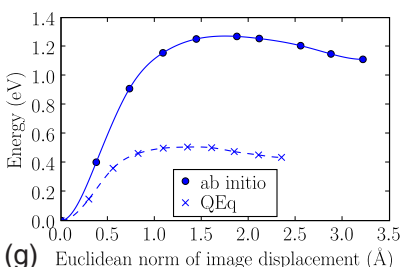

(g) Euclidean norm of image displacement $(\AA)$
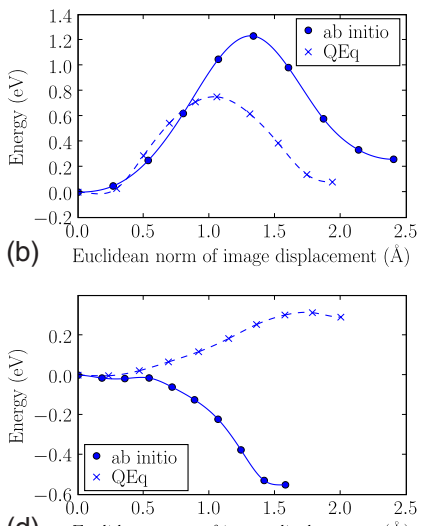

(d) Euclidean norm of image displacement $(\AA)$
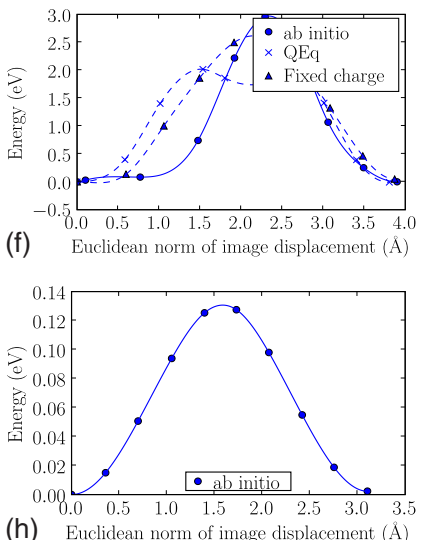

(h) Euclidean norm of image displacement $(\AA)$
FIG. 2. (Color online) Transition pathway graphs of energy versus reaction coordinate for (a) Ti upper hollow to Ti lower hollow, (b) Ti-iI to Ti-iII, (c) O-II to O-II, (d) TiO-IV to TiO-III, and (e) TiO-II to TiO-I, (f) $\mathrm{TiO}_{2}-\mathrm{I}$ to $\mathrm{TiO}_{2}-\mathrm{Ib}$ and (g) $\mathrm{TiO}_{2}-\mathrm{I}$ to $\mathrm{TiO}_{2}$-II, and (h) $\mathrm{O}_{2}$-I to $\mathrm{O}_{2}$-Ia. The energy is measured in $\mathrm{eV}$ relative to the starting configuration, while the reaction coordinate is measured as the Euclidean norm of the displacement of each system image from the initial configuration as measured in angstroms.

term, using only a repulsive Buckingham potential, it is not possible to obtain the O-I defect state with the QEq potential without some modification to account for the $\mathrm{O}-\mathrm{O}$ attractive interaction.

The second site, O-II, has the oxygen atom adsorbed midway between two rows of two-coordinated surface oxygen atoms. The O-II site is located slightly off-center above a five-coordinated surface titanium atom. It is displaced along the $\left[\begin{array}{lll}0 & 0 & 1\end{array}\right]$ direction by $0.62 \AA$ and is shown in Fig. 4(b). The QEq model, on the other hand, predicts that this O-II adsorption site is actually centered precisely above the fivecoordinated surface titanium atom. The relative energies of these binding sites, as calculated by DFT and the QEq model, are given in Table I.

The O-II configuration can be formed from an O-I adatom by pulling one of the two oxygen atoms within the adsorbed dioxygen unit away from the two-coordinated oxygen row and into the middle of the trench as shown in Fig. 4(a). Once in the O-II adsorption site, the oxygen atom can translate along the $\left[\begin{array}{lll}0 & 0 & 1\end{array}\right]$ direction, to the neighboring equivalent O-II sites, as shown in Fig. 4(b). The O-II-IIa transition in the direction of the nearest five-coordinated surface titanium 


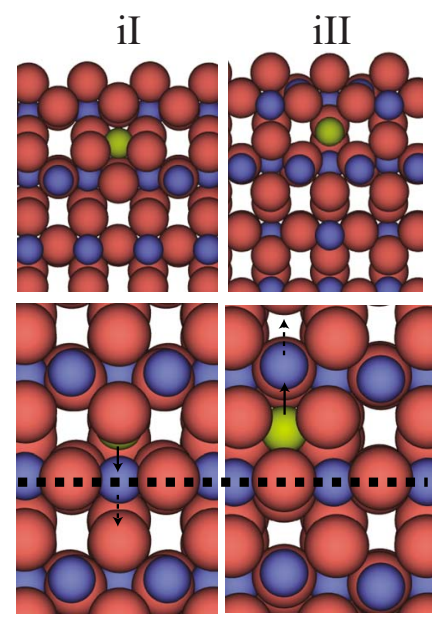

FIG. 3. (Color online) The diagram shows titanium interstitial site I (left) and titanium interstitial site II (right). The color scheme is the same as in previous diagrams. The interstitial titanium atom is shown in yellow. The top diagrams show the interstitial sites from along the $[1 \overline{1} 0]$ direction. The bottom diagrams show the interstitial sites viewed from directly above the (llll 100$)$ surface. In the bottom diagrams, the horizontal dashed line indicates the row of surface two-coordinated atoms. In the bottom left figure, the arrows indicate the movement of atoms during the Ti-iI to Ti-upper hollow transition via the replacement mechanism. The solid arrow indicates the movement of the initial interstitial atom, while the dotted arrow indicates the movement of the replaced titanium atom, which becomes the final adsorbed atom. In the bottom right figure, similar arrows indicate the movement of atoms during the Ti-iII to Ti-lower hollow transition via the replacement mechanism.

atom has a barrier of $0.29 \mathrm{eV}$. The O-II-IIb transition in the opposite direction has a negligible barrier (less than 0.02 $\mathrm{eV})$. Combined, these two transitions give an effective DFT transition barrier of $0.29 \mathrm{eV}$ for translation of the $\mathrm{O}$ adatom along the $\left[\begin{array}{lll}0 & 0 & 1\end{array}\right]$ direction. Because the QEq model predicts that the O-II adsorption site is directly above a surface fivecoordinated titanium atom, rather than off-center, the same effective transition can occur with only a single step. The energy profile for this combined transition is shown in Fig. 2(c) with the barrier significantly higher than the DFT one.

An adsorbed oxygen atom in the O-I site can move to an adjacent O-I site by dissociating from the dioxygen unit, moving along the $\left[\begin{array}{lll}0 & 0 & 1\end{array}\right]$ axis, and bonding with a neighboring two-coordinated oxygen atom within the same row (2.5 $\mathrm{eV}$ ), as shown in Fig. 4(a). The activation barriers as calculated by DFT and the QEq model are given in Table II.

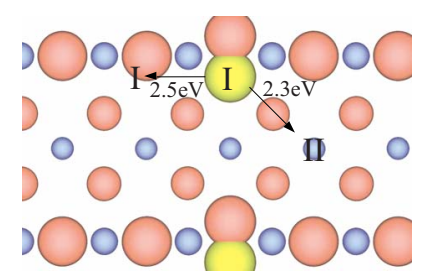

(a)

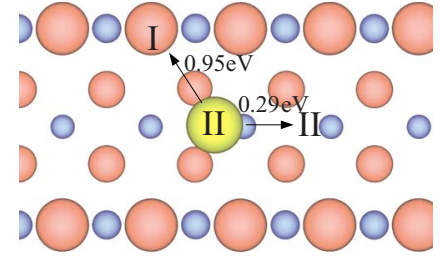

(b)

$$
1.44 \mathrm{eV}
$$

FIG. 4. (Color online) (a) shows the oxygen adsorption site I, while (b) shows the oxygen adsorption site II. Both figures are viewed from directly above the (110) surface. The color scheme is the same as in previous diagrams. The horizontal rows of larger oxygen atoms represent the surface rows of two-coordinated oxygen atoms. The arrows represent the various transitions available to the adatoms in these adsorption sites. The energies correspond to the DFT activation barriers for each transition.

\section{TiO adcluster}

The TiO cluster has four unique binding sites on the rutile (110) surface shown in Fig. 5. The relative energies of these sites as calculated by both DFT and the QEq model are shown in Table I. The TiO-I site has the titanium atom located in the upper hollow site, while the oxygen atom is located above a five-coordinated surface titanium atom. The TiO-II site also has the titanium atom situated in the upper hollow site, but in this case the cluster oxygen atom is pointed up, away from the rutile surface. Again the QEq model is seen to favor the site with the $\mathrm{O}$ atom pointing away from the surface while the DFT results indicate the opposite result. The relative energies of these binding sites, as calculated by DFT and the QEq model, are given in Table I.

According to DFT, the TiO-II was calculated to have an energy $0.61 \mathrm{eV}$ above the TiO-I site; however, the QEq model predicts that this configuration has an energy $0.24 \mathrm{eV}$ below that of the TiO-I site. The TiO-III site has the cluster titanium atom located in the lower hollow site, while the cluster oxygen atom is located above a five-coordinated surface titanium atom. The final structure, TiO-IV, has the cluster titanium atom located in a lower hollow site, with the oxygen pointed up away from the rutile surface.

As shown in Fig. 5(a), the TiO-I cluster can move into an equivalent TiO-I binding site simply by shifting the cluster oxygen atom from one five-coordinated surface titanium atom to the next one over. The TiO-I cluster can also shift into the TiO-II binding configuration by allowing the cluster oxygen atom to move up away from the surface. As shown in

TABLE III. The bond lengths, Bader atomic charges, Bader atomic spins, and the Laplacian of the electronic charge density at the bond critical points (BCPs) are listed here for $\mathrm{O}_{2}$ in vacuum, the $\mathrm{O}_{2}$-I admolecule, and the O-I dioxygen unit.

\begin{tabular}{lcccc}
\hline \hline System & $\begin{array}{c}\text { O-O bond length } \\
(\AA)\end{array}$ & Bader charge $(e)$ & Bader spin & $\nabla^{2} \rho_{\text {BCP }}\left(e a_{0}^{-5}\right)$ \\
\hline $\mathrm{O}_{2}$ in vacuum & 1.234 & 0.0 & 0.5 & -0.33 \\
$\mathrm{O}_{2}$-I dioxygen & 1.225 & $0.08,-0.06$ & $0.52,0.45$ & -0.39 \\
O-I dioxygen & 1.416 & -0.42 & 0.0 & 0.24 \\
\hline \hline
\end{tabular}




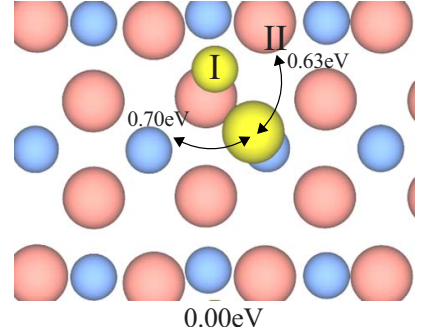

(a)

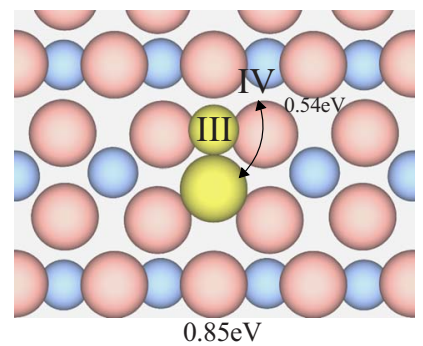

(c)

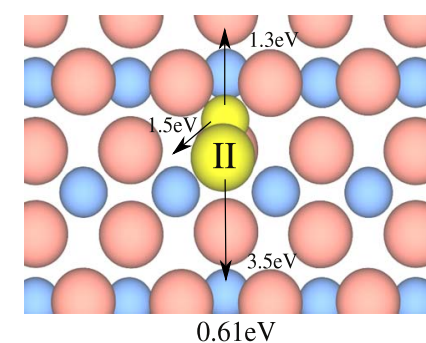

(b)

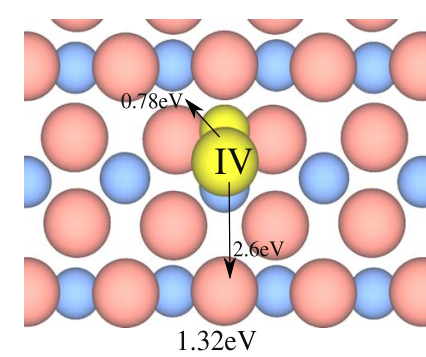

(d)

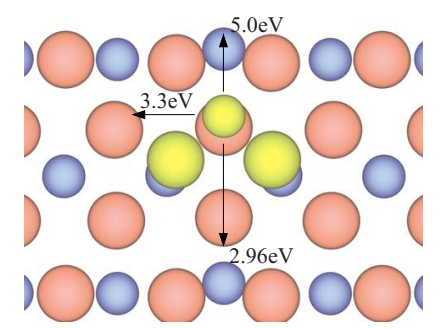

(a)

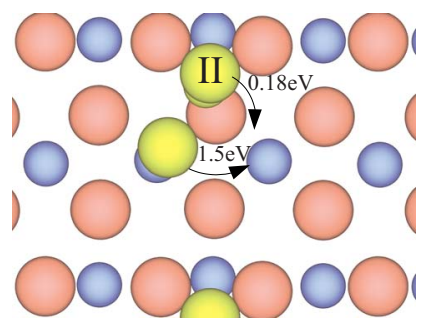

(b)

$1.1 \mathrm{eV}$

FIG. 6. (Color online) (a) The $\mathrm{TiO}_{2}$ adsorption site I. (b) The $\mathrm{TiO}_{2}$ adsorption site II. Both figures are viewed from directly above the (110) surface. The color scheme is the same as in previous diagrams. The horizontal rows of larger oxygen atoms represent the surface rows of two-coordinated oxygen atoms. The arrows represent the various transitions available to the $\mathrm{TiO}_{2}$ clusters in these adsorption sites. The energies correspond to the DFT activation barriers for each transition.

\section{E. $\mathrm{TiO}_{2}$ adcluster}

FIG. 5. (Color online) (a) The TiO adsorption site I. (b) The TiO adsorption site II. (c) The TiO adsorption site III. (d) The TiO adsorption site IV. All figures are viewed from directly above the (110) surface. The color scheme is the same as in previous diagrams. The horizontal rows of larger oxygen atoms represent the surface rows of two-coordinated oxygen atoms. The arrows represent the various transitions available to the $\mathrm{TiO}$ clusters in these adsorption sites. The energies correspond to the DFT activation barriers for each transition.

Fig. 5(c), the TiO-III configuration can change to the TiO-IV configuration by similarly lifting the cluster oxygen atom away from the rutile surface.

The following transitions are shown in Fig. 5(b). The TiO cluster can hop from the TiO-II to the TiO-IV binding site $(1.5 \mathrm{eV})$ similarly to the Ti upper hollow-lower hollow transition mentioned previously. The TiO-II adcluster can also hop into a neighboring equivalent TiO-II site via two pathways. The first pathway, TiO-II-IIa, has the titanium atom hopping over the two-coordinated oxygen row onto the opposite side of the same row along the $[1 \overline{1} 0]$ direction. The second pathway, TiO-II-IIb, has the titanium atom hopping in the opposite direction along the $[1 \overline{1} 0]$ direction to bind alongside a neighboring two-coordinated oxygen row. Barriers for these transitions can be found in Table II.

The following transitions are shown in Fig. 5(d). The TiO-IV cluster can hop to an adjacent TiO-IV site in a similar way to the TiO-II-IIb transition. The cluster hops from one two-coordinated oxygen row to a neighboring row across the trench in the $[1 \overline{1} 0]$ direction. During all of the transitions involving the TiO-II and TiO-IV adcluster, the oxygen atom stays pointed essentially in the opposite direction from the three surface oxygen atoms to which the adcluster titanium is bound. There is reasonable agreement for the barriers between DFT and QEq, except for the TiO-II to TiO-I transition, and the TiO-III to TiO-IV transition, where the relative magnitudes of the barriers are reversed (see Table II).

The $\mathrm{TiO}_{2}$ cluster is composed of a central titanium atom, which is bound to two oxygen atoms in a bent triatomic geometry. The isolated cluster has an O-Ti-O bond angle of $108.5^{\circ}$.

The $\mathrm{TiO}_{2}$ cluster has two unique binding sites on the rutile (110) surface. The first binding site, $\mathrm{TiO}_{2}-\mathrm{I}$, has the titanium atom located in an upper hollow adatom binding site with the two cluster oxygen atoms resting almost directly above two five-coordinated surface titanium atoms. This site, shown in Fig. 6(a), has a net DFT binding energy of $-6.51 \mathrm{eV}$ relative to the free cluster in vacuum and the perfect surface. The second binding site, $\mathrm{TiO}_{2}$-II, is similar to $\mathrm{TiO}_{2}-\mathrm{I}$ except one of the cluster oxygen atoms is pointed upward directly away from the rutile surface. It is shown in Fig. 6(b). The relative energies of these binding sites as calculated by DFT, QEq model, and the Matsui fixed-charge model are given in Table I.

As shown in Fig. 6(a), the $\mathrm{TiO}_{2}-\mathrm{I}$ adcluster can move into equivalent $\mathrm{TiO}_{2}$-I sites in three different ways. In the $\mathrm{TiO}_{2}$-I-Ia transition, the adcluster essentially translates along the $\left[\begin{array}{lll}0 & 0 & 1\end{array}\right]$ direction into a neighboring $\mathrm{TiO}_{2}-\mathrm{I}$ binding site.

In the $\mathrm{TiO}_{2}-\mathrm{I}-\mathrm{Ib}$ transition, the adcluster titanium atom moves across a trench in the [1 $1 \overline{0} 0]$ direction ending at an equivalent binding site on a neighboring two-coordinated oxygen atom row. During the course of this transition, the adcluster $\mathrm{O}-\mathrm{Ti}-\mathrm{O}$ angle increases to $180^{\circ}$ at the transition midpoint, then decreases to its original value at the final state (which is equivalent to the initial state). The QEq model predicts that the midpoint of this transition is itself an energy minimum. This is probably due to the fact that the geometry at this midpoint places the two oxygen atoms above two five-coordinated surface titanium atoms and the central titanium atom between two surface oxygen atoms. Electrostatically, this is favorable for the QEq model which, unlike DFT, does not take into account the bent bond angle preference of the $\mathrm{TiO}_{2}$ unit. Thus, although the $\mathrm{TiO} 2$ binding to the surface can be understood in terms of electrostatics, the transitions cannot be, as electrostatics would not predict a bent $\mathrm{TiO}_{2}$ unit. 
In the $\mathrm{TiO}_{2}$-I-Ic transition, the adcluster titanium atom hops over the two-coordinated oxygen row in the [1 $1 \overline{1} 0]$ direction into an equivalent site on the opposite side of the same row. Throughout this transition, the two adcluster oxygen atoms are lifted up away from the surface and move in a semicircular pattern finally ending almost directly above two five-coordinated titanium atoms on the other side of the twocoordinated oxygen row as shown in Fig. 2(f).

Also shown in Fig. 6(a), the $\mathrm{TiO}_{2}$-I adcluster can move into the $\mathrm{TiO}_{2}$-II binding position by essentially rotating about one of its Ti-O bond axes. The other adcluster oxygen atom then moves up, away from the rutile surface until it is located almost directly above the adcluster titanium atom, pointed almost directly away from the rutile surface, as shown in Fig. $2(\mathrm{~g})$.

As shown in Fig. 6(b), the $\mathrm{TiO}_{2}$-II adcluster can move into an equivalent $\mathrm{TiO}_{2}$-II binding site in one of two different ways. During the $\mathrm{TiO}_{2}$-II-IIa transition, the adcluster essentially rotates about the Ti-O axis that is pointed away from the surface. In this way, the lower adcluster oxygen atom moves from being directly above one five-coordinated surface titanium atom to the neighboring five-coordinated surface titanium atom. The adcluster titanium and the other oxygen atom do not shift very much from their initial positions.

During the $\mathrm{TiO}_{2}$-II-IIb transition, the adcluster titanium hops in the [1ㅣㅣㄹ direction, over the two-coordinated oxygen atom row, into an equivalent binding position on the opposite side of the same two-coordinated oxygen row. During this process, the adcluster oxygen atom initially pointed away from the surface binds to a five-coordinated surface titanium atom on the other side of the two-coordinated oxygen row. Also, the other adcluster oxygen, initially bound to a fivecoordinated surface titanium, ends up pointed away from the rutile surface. The activation barriers as calculated by DFT, the QEq model, and the fixed-charge Matsui model are given in Table II. For the $\mathrm{TiO}_{2}$ cluster, the QEq model gives lower energy barriers than both the DFT and the fixed-charge model. This is mainly due to an underestimate of the charge on the $\mathrm{TiO}_{2}$ cluster in the QEq model.

\section{F. $\mathrm{O}_{2}$ adcluster}

The $\mathrm{O}_{2}$ adcluster has one binding site of interest illustrated in Figs. 7(a) and 7(b). Other binding configurations were found, but they were calculated to be at least $2 \mathrm{eV}$ above this minimum energy configuration and will not be discussed here. In the $\mathrm{O}_{2}$-I binding site, one of the dioxygen atoms bound to a five-coordinated titanium atom within the rutile surface, with the other oxygen atom pointed somewhat away from the surface, such that the O-O-Ti bond angle is $126.5^{\circ}$. This binding site has a net DFT binding energy of $0.49 \mathrm{eV}$ relative to the isolated dioxygen molecule and the perfect surface. The O-O distance within the adsorbed molecule is $1.225 \AA$, which compares well with the isolated $\mathrm{O}_{2}$ bond distance of $1.234 \AA$. A Bader analysis (see Table III) indicated that the upper of the two $\mathrm{O}_{2}$ oxygen atoms had an atomic charge of $0.08 e$, while the lower oxygen atom had a charge of $-0.06 e$. The atomic spin densities of the two atoms were 0.52 and 0.45 , respectively. The Laplacian of the elec-

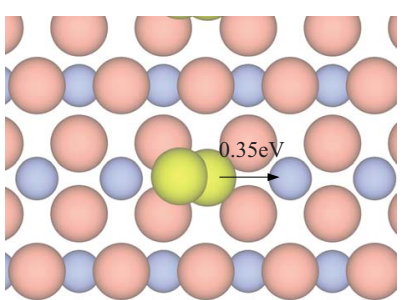

(a)

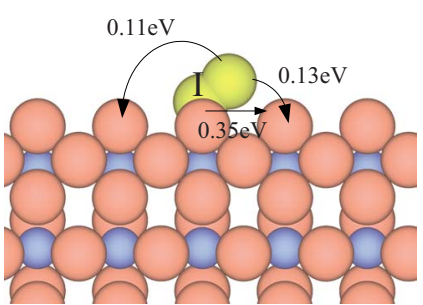

(b)
FIG. 7. (Color online) (a) and (b) show the $\mathrm{O}_{2}$ adsorption site. (a) shows it from the $\left[\begin{array}{lll}1 & 1 & 0\end{array}\right]$ direction, while (b) shows it from the $\left[\begin{array}{lll}0 & 0 & 1\end{array}\right]$ direction. The color scheme is the same as in previous diagrams. The horizontal rows of larger oxygen atoms in (a) represent the surface rows of two-coordinated oxygen atoms. The arrows represent the various transitions available to the $\mathrm{O}_{2}$ cluster. The energies correspond to the DFT activation barriers for each transition.

tronic charge density at the $\mathrm{O}-\mathrm{O}$ bond critical point was -0.39 . All of these values compare quite well with the corresponding values calculated for the isolated $\mathrm{O}_{2}$ molecule with the same DFT methodology. This provides good evidence that the adsorbed $\mathrm{O}_{2}$ admolecule in site O-I can be considered chemically similar to the $\mathrm{O}_{2}$ gas molecule. Thus, although the surface bonding of the $\mathrm{O}_{2}$ admolecule is weak there is a small amount of charge transfer that would give rise to some ionic bonding. There may also be some element of covalency but with such weak bonding it is not possible to determine this.

Unfortunately, no calculations could be performed on adsorbed $\mathrm{O}_{2}$ using either the QEq potential or the Matsui potential. The QEq potential will not model this system properly, because it lacks an attractive O-O potential of any kind, instead relying only on a repulsive Buckingham potential to keep oxygen atoms from closely approaching each other. This means that $\mathrm{O}_{2}$ cannot be formed with this potential, either in isolation or adsorbed onto a surface. The Matsui potential cannot be used here either because the system is not stoichiometric. The relative energies of these binding sites as calculated by DFT are given in Table I.

As shown in Fig. 7(b), the $\mathrm{O}_{2}$ cluster can hop along the [0 0 1] direction from one binding site to the next equivalent site via a two-step process. During the first step, $\mathrm{O}_{2}$-I-Ia, the upper adcluster oxygen atom bends toward the surface and bonds with a neighboring five-coordinated surface titanium atom, while the lower adcluster oxygen atom breaks its bond with the underlying five-coordinated titanium atom and moves up away from the surface (in effect becoming the upper oxygen atom on the new adsorption site). In this way, the final state has the adcluster pointing at an equivalent $126.5^{\circ}$ angle from the surface but in the opposite direction along the $\left[\begin{array}{lll}0 & 0 & 1\end{array}\right]$ direction. This transition has a barrier of $0.13 \mathrm{eV}$ as calculated in Fig. 2(h) with the transition described in Fig. 7(b). The second half of the two-step process, $\mathrm{O}_{2}-\mathrm{I}-\mathrm{Ib}$, is a simple bend of the upper adcluster oxygen atom such that the dioxygen molecule is bent in the original direction (before the $\mathrm{O}_{2}$-I-Ia transition occurred). By alternating between transitions $\mathrm{O}_{2}-\mathrm{I}-\mathrm{Ia}$ and $\mathrm{O}_{2}-\mathrm{I}-\mathrm{Ib}$, the adsorbed dioxygen can essentially "tumble" along the center of the surface trench in the $\left[\begin{array}{lll}0 & 0 & 1\end{array}\right]$ direction. The activation barriers as calculated by DFT are given in Table II. 
A second mechanism for $\mathrm{O}_{2}$ diffusion involves a similar procedure except that the first step occurs somewhat differently. In this $\mathrm{O}_{2}$-I-Ic transition, shown in Figs. 7(a) and 7(b), the bottom oxygen atom in the adsorbed dioxygen instead hops from one five-coordinated titanium to the next, while the upper oxygen atom shifts position only slightly. By alternating between the $\mathrm{O}_{2}$-I-Ic and $\mathrm{O}_{2}$-I-Ib transitions, the dioxygen molecules can "walk" along the center of the surface trench in the $\left[\begin{array}{lll}0 & 0 & 1\end{array}\right]$ direction. This has an energy barrier of $0.35 \mathrm{eV}$ shown in Fig. 7(a).

\section{G. Atomic charges}

Atomic charges were calculated using the densities obtained from the DFT calculations for comparison with the atomic charges calculated from the QEq scheme. The QEq was found to generally overestimate the charge transfer for atoms in the adsorbed clusters as well as the surfaces and the bulk. For example, the bulk oxygen charge was calculated to be -0.92 according to the DFT Bader analysis and -1.29 according to the QEq scheme. Within the TiO-I system, the adcluster oxygen atom has a Bader charge of -0.80 , while the QEq scheme gives a charge of -0.93 . The TiO-I titanium atom has a Bader charge of 0.98 , while the QEq scheme gives a charge of 2.01. The bridging oxygen nearest to the adcluster has a Bader charge of -0.98 and a QEq charge of -1.20 .

The oxygen charge discrepancy between the Bader and QEq charges amounts to 0.37 for the bulk, 0.22 for the bridging oxygen nearest the TiO-I adcluster, and 0.13 for the adcluster oxygen. Generally, the higher the coordination of the atom, the worse the QEq scheme approximates its Bader charge. This implies that the charge at the minimum of the self-energy function is nearly correct, but the hardness is too small, resulting in a larger charge transfer than the DFT predicts. These differences could be alleviated by increasing the hardness of the titanium or oxygen atoms within the QEq model, while adjusting the electronegativity to keep the minimum of the self-energy function constant.

\section{CONCLUSIONS}

Using three different models, we have examined the binding sites and mobility of small titanium oxide clusters adsorbed onto the rutile (110) surface. Unconstrained geometry optimizations were used to find a set of adsorption sites for each of the small clusters studied: $\mathrm{Ti}, \mathrm{O}, \mathrm{TiO}, \mathrm{O}_{2}$, and $\mathrm{TiO}_{2}$. The nudged elastic band method was then used to obtain the transition pathways between these adsorption sites giving geometric and energetic descriptions of various diffusion pathways.

Generally, the diffusion barriers for adsorbed clusters are very high. This would indicate that impacting clusters, from a magnetron sputtering process, for example, would require very high local temperatures and energies in order to diffuse along a pristine surface. The exception to this is the $\mathrm{O}_{2}$ cluster, which should be relatively mobile along the [001] direction even at room temperature. Additionally, none of the cluster adsorption sites are "bulklike." That is, in each mini- mum energy configuration, none of the cluster atoms are near their bulk positions. The exception to this is the adsorbed oxygen atom, which has a higher energy adsorption site nearly above a surface five-coordinated titanium atom.

Agreement between the QEq results and the ab initio results was generally quite good (see Table I). The QEq model matched the DFT results particularly well for the energetics of adsorbed and interstitial $\mathrm{Ti}$ atoms. In this case, it not only predicted the correct energetic ordering of the four systems but also did a fairly good job at predicting their relative energies. The QEq model accurately predicts that the two upper hollow $\mathrm{TiO}$ adsorption sites are more energetically favorable than the two lower hollow adsorption sites; however, the QEq inaccurately predicts that site TiO-II is more favorable than TiO-I and that TiO-IV is more favorable than TiOIII. It can be concluded that the QEq model predicts that the oxygen within this cluster is more repelled by the rutile surface than the DFT calculations would indicate. The QEq did predict the correct energetic ordering of the two $\mathrm{TiO}_{2}$ adcluster systems. However, it underestimated the relative energetics by about $65 \%$, while the Matsui fixed-charge potential was nearly exact with respect to the DFT calculations. Most of the minimum energy configurations found using DFT were also found using the $\mathrm{QEq}$ potential, with the exception of the O-I site, and the sites involving the adsorbed $\mathrm{O}_{2}$ molecule. This suggests that a modification to the QEq model to include an attractive $\mathrm{O}-\mathrm{O}$ interaction is necessary to describe surface processes. The resulting speed and accuracy would allow molecular-dynamics simulations of sputtering including individual deposition events and subsequent surface diffusion and reactions.

The QEq transition barrier results also agree quite well with the DFT results, with most of the QEq barriers lying within $0.5 \mathrm{eV}$ of the corresponding DFT barrier (see Table II). In general, the QEq tends to underestimate the transition barriers more often than it overestimates them and by larger amounts. The QEq model did underestimate the Ti interstitial formation barriers by more than $1 \mathrm{eV}$, which would lead to a prediction of excess Ti interstitial defects during MD simulations at lower energies than expected by DFT. Although the QEq model underestimated every TiO barrier except for two of them, the largest deviation between the QEq and DFT predictions was only $0.54 \mathrm{eV}$ with most deviations less than $0.4 \mathrm{eV}$. The QEq model did not work as well for the $\mathrm{TiO}_{2}$ transition barriers, however. All of these barriers were underestimated, with three barriers being underestimated by more than $1 \mathrm{eV}$. However, most of these barriers are so high that the transitions are nearly inaccessible except at very high temperatures. One point of concern, however, is that the QEq potential underestimates the $\mathrm{TiO}_{2}-\mathrm{I}$ to $\mathrm{TiO}_{2}-\mathrm{II}$ and $\mathrm{TiO}_{2}-\mathrm{II}$ to $\mathrm{TiO}_{2}$-IIa transitions by enough to effectively make them accessible at room temperature, whereas their DFT energy barriers are both well over $1 \mathrm{eV}$. This means that more instances of the $\mathrm{TiO}_{2}$-II configuration will be visible in any MD simulations done using this QEq potential than with DFT-MD, for example.

Bond, charge, and spin analysis using Bader's atoms in molecules approach indicates that the adsorbed $\mathrm{O}_{2}$ molecule closely resembles the isolated $\mathrm{O}_{2}$ molecules in vacuum. A similar analysis performed on the apparent " $\mathrm{O}_{2}$ " unit found 
at the O-I adsorption site shows that this $\mathrm{O}_{2}$ unit is significantly different from an isolated $\mathrm{O}_{2}$ molecule.

It would be expected in most growth processes that $\mathrm{O}_{2}$ dimers would be a prevalent activating species. Because of the high diffusion barriers and hence the general immobility of adsorbed and interstitial atoms and clusters, the indication is that the surface growth would be dominated by the mobility of the $\mathrm{O}_{2}$ dimers over the surface.

Comparison of the $\mathrm{QEq}$ charges with those obtained through a Bader analysis indicates that the QEq generally overestimates charge transfer within rutile bulk, surfaces, and adsorbed clusters. The higher the coordination of the atom, the greater the overestimation tends to be. This indicates that the hardness of the atoms is too small. Upward adjustment of the QEq hardness, and simultaneous adjustment of the electronegativity in order to keep the self-energy function minimum constant, may result in better energetic and transition barrier results from this method.

\section{ACKNOWLEDGMENTS}

The authors gratefully acknowledge funding for this research acquired from the EPSRC-GB through Grants No. EP/C524322/1 and No. EP/C524349. *maes@lboro.ac.uk

†y.moghaddam@reading.ac.uk

tpaul.mulheran@strath.ac.uk

${ }^{1}$ D. Cronemeyer, Phys. Rev. 87, 876 (1952).

${ }^{2}$ M. K. Nazeeruddin, T. Bessho, L. Cevey, S. Ito, C. Klein, F. D. Angelis, S. Fantacci, P. Comte, P. Liska, H. Imai, and M. Graetzel, J. Photochem. Photobiol. Chem. 185, 331 (2007).

${ }^{3}$ S. Takeda, S. Suzuki, H. Odaka, and H. Hosono, Thin Solid Films 392, 338 (2001).

${ }^{4}$ D. Dumitriu, A. R. Bally, C. Ballif, P. Hones, P. E. Schmid, R. Sanjins, F. Lvy, and V. I. Prvulescu, Appl. Catal. Environ. 25, 83 (2000).

${ }^{5}$ A. J. Nozik, Annu. Rev. Phys. Chem. 29, 189 (1978).

${ }^{6}$ R. Hummel and K. Guenther, Handbook of Optical Properties: Thin Films for Optical Coatings (CRC, Boca Raton, FL, 1995), Vol. 1.

${ }^{7}$ T. Watanabe, A. Nakajimaa, R. Wang, M. Minabea, S. Koizumia, A. Fujishimab, and K. Hashimoto, Thin Solid Films 351, 260 (1999).

${ }^{8}$ P. Zeman and S. Takabayashi, J. Vac. Sci. Technol. 20, 388 (2002).

${ }^{9}$ K. Okimura, Surf. Coat. Technol. 135, 286 (2001).

${ }^{10}$ U. Diebold, Surf. Sci. Rep. 48, 53 (2003).

${ }^{11}$ H. Onishi and Y. Iwasawa, Phys. Rev. Lett. 76, 791 (1996).

${ }^{12}$ R. D. Smith, R. A. Bennett, and M. Bowker, Phys. Rev. B 66, 035409 (2002).

${ }^{13}$ S. Wendt, P. Sprunger, E. Lira, G. Madsen, J. H. Z. Li, J. Matthiesen, A. Blekinge-Rasmussen, E. Lgsgaard, B. Hammer, and
F. Besenbacher, Science 320, 1755 (2008).

${ }^{14}$ H. Jonsson, G. Mills, and K. W. Jacobsen, Classical and Quantum Dynamics in Condensed Phase Simulations (International School of Physics, Villa Marigola, 1997).

${ }^{15} \mathrm{G}$. Henkelman and H. Jonsson, J. Chem. Phys. 113, 9978 (2000).

${ }^{16}$ G. Henkelman, B. P. Uberuaga, and H. Jonsson, J. Chem. Phys. 113, 9901 (2000).

${ }^{17}$ S. D. Kenny, A. P. Horsfield, and H. Fujitani, Phys. Rev. B 62, 4899 (2000).

${ }^{18}$ C. Hartwigsen, S. Goedecker, and J. Hutter, Phys. Rev. B 58, 3641 (1998).

${ }^{19}$ A. Hallil, R. Tetot, F. Berthier, I. Braems, and J. Creuze, Phys. Rev. B 73, 165406 (2006).

${ }^{20}$ V. Swamy and J. D. Gale, Phys. Rev. B 62, 5406 (2000).

${ }^{21}$ V. Swamy, J. Gale, and L. Dubrovinsky, J. Phys. Chem. Solids 62, 887 (2001).

${ }^{22}$ E. Demiralp, T. Cagin, and W. A. Goddard, Phys. Rev. Lett. 82, 1708 (1999).

${ }^{23}$ A. Rappe and W. Goddard, J. Phys. Chem. 95, 3358 (1991).

${ }^{24}$ C. Noguera, Physics and Chemistry at Oxide Surfaces (Cambridge University Press, Cambridge, 1996).

${ }^{25}$ M. Matsui and M. Akaogi, Mol. Simul. 6, 239 (1991).

${ }^{26} \mathrm{R}$. Bader, Atoms in Molecules: A Quantum Theory (Clarendon Press, Oxford, 1994).

${ }^{27}$ E. Sanville, S. Kenny, R. Smith, and G. Henkelman, J. Comput. Chem. 28, 899 (2007). 